\title{
BOUNDED POINT EVALUATIONS AND SMOOTHNESS PROPERTIES OF FUNCTIONS IN $R^{p}(X)\left(^{(}\right)$ \\ BY \\ EDWIN WOLF
}

\begin{abstract}
Let $X$ be a compact subset of the complex plane $\mathbf{C}$. We denote by $R_{0}(X)$ the algebra consisting of the (restrictions to $X$ of) rational functions with poles off $X$. Let $m$ denote 2-dimensional Lebesgue measure. For $p>1$, let $L^{p}(X)=L^{p}(X, d m)$. The closure of $R_{0}(X)$ in $L^{p}(X)$ will be denoted by $R^{p}(X)$. Whenever $p$ and $q$ both appear, we assume that $1 / p+1 / q=1$.

If $x$ is a point in $X$ which admits a bounded point evaluation on $R^{p}(X)$, then the map which sends $f$ to $f(x)$ for all $f \in R_{0}(X)$ extends to a continuous linear functional on $R^{p}(X)$. The value of this linear functional at any $f \in R^{p}(X)$ is denoted by $f(x)$. We examine the smoothness properties of functions in $R^{P}(X)$ at those points which admit bounded point evaluations. For $p>2$ we prove in Part I a theorem that generalizes the "approximate Taylor theorem" that James Wang proved for $R(X)$.

In Part II we generalize a theorem of Hedberg about the convergence of a certain capacity series at a point which admits a bounded point evaluation. Using this result, we study the density of the set $X$ at such a point.
\end{abstract}

\section{PART I. SMOOTHNESS PROPERTIES OF FUNCTIONS IN $R^{p}(X)$}

Let $X$ be a compact subset of the complex plane C. We denote by $R_{0}(X)$ the algebra consisting of the (restrictions to $X$ ) of rational functions with poles off $X$. Let $m$ denote 2-dimensional Lebesgue measure. For $p \geqslant 1$, let $L^{p}(X)=L^{p}(X, d m)$. The closure of $R_{0}(X)$ in $L^{p}(X)$ will be denoted by $R^{p}(X)$. Whenever $p$ and $q$ both appear, we will assume that $1 / p+1 / q=1$.

\section{Bounded point derivations.}

Definition (1.1). For $x \in X$ we say that $x$ admits a bounded point derivation of order $s$ on $R^{p}(X)$ if there exists a constant $C$ such that $\left|f^{(s)}(x)\right| \leqslant$ $C\|f\|_{p}$ for all $f \in R_{0}(X)$.

When $x$ admits a bounded point derivation of order $s$ on $R^{p}(X)$, the map $f \mapsto f^{(s)}(x) / s$ ! extends from $R_{0}(X)$ to a bounded linear functional on $R^{p}(X)$.

Received by the editors July $13,1976$.

AMS (MOS) subject classifications (1970). Primary 46-XX, 46EXX, 46E99.

Key words and phrases. Rational functions, compact set, $L^{p}$-spaces, bounded point evaluation, representing function, admissible function, full area density, $\Gamma_{q}$-capacity.

(')This paper contains results of the author's doctoral dissertation. The author would like to thank Andrew Browder, his advisor, for his suggestions and comments. He is also grateful to John Wermer and James Wang for helpful conversations. 
We denote this bounded linear functional by $D_{x}^{s}$.

Definition (1.2). When $x$ admits a bounded point derivation of order 0 , we say that $x$ admits a bounded point evaluation. For $f \in R^{p}(X)$ we define $f(x)=D_{x}^{0} f$.

Definition (1.3). For each $p \geqslant 2$ the inner set for $R^{p}(X)$ is the set of points in $X$ which admit bounded point evaluations, and we denote it by $S^{p}(X)$.

Proposition (1.1). For each $p \geqslant 2, S^{p}(X)$ is an $F_{\sigma}$ set.

Proof. Write $S^{p}(X)=\bigcup_{n=1}^{\infty} S_{n}^{p}(X)$ where

$$
S_{n}^{p}(X)=\left\{x \in X|| f(x) \mid \leqslant n\|f\|_{p} \text { for all } f \in R^{p}(X)\right\} .
$$

We show that each set $S_{n}^{p}(X)$ is closed. Suppose that $\left\{x_{k}\right\} \subset S_{n}^{p}(X)$ and that $x_{k} \rightarrow x \in X$. Let $L_{x_{k}} f=f\left(x_{k}\right)$ and observe that the $L_{x_{k}}$ are a family of linear functionals bounded in norm by $n$. Since $L_{x_{k}} f \rightarrow f(x)$ for $f \in R_{0}(X)$, and $R_{0}(X)$ is dense in $R^{p}(X)$, it follows that $x \in S_{n}^{p}(X)$. Thus each $S_{n}^{p}(X)$ is closed.

2. Potentials and representing functions. In this paper $z$ will denote the identity function.

Definition (2.1). Let $\psi$ be a positive nondecreasing function on $(0, \infty)$. For each $g \in L^{q}(X), q \geqslant 1$, we define the $\psi$-potential of $g, U_{g}^{\psi}$, by

$$
U_{g}^{\psi}(y)=\int \frac{|g|}{\psi(|z-y|)} d m .
$$

If $1 / \psi(|z|)$ is locally summable with respect to $m$, Fubini's theorem implies that $U_{g}^{\psi}$ is locally summable; hence $U_{g}^{\psi}<\infty$ a.e. $(m)$.

Definition (2.2). When $\psi(r)=r$, we denote $U_{g}^{\psi}$ by $\tilde{g}$.

Definition (2.3). When $\psi(r)=r^{q}, 1<q<2$, we denote $U_{g}^{\psi}$ by $U_{g}^{q}$.

Definition (2.4). We define the Cauchy transform of $g$ to be

$$
\hat{g}(y)=\int(z-y)^{-1} g d m \text { for all } y \text { where } \hat{g}(y)<\infty .
$$

For the proof of the following lemma we refer the reader to Sinanjan [16] or Brennan [1, pp. 10-11]. Brennan's proof uses the Cauchy transform.

LEMMA (2.1). Let $X \subset \mathbf{C}$ be compact and have no interior. Then $R^{p}(X)=$ $L^{p}(X)$ for $1<p<2$.

It follows from the Riesz representation theorem that if $x \in S^{p}(X)$, then there is a $g \in L^{q}(X)$ such that $f(x)=\int f g d m$ for all $f \in R^{p}(X)$. We call such a $g$ a representing function for $x$. If $R^{p}(X) \neq L^{p}(X)$, there is a nonzero function $g \in L^{q}(X)$ such that $\int f g d m=0$ for all $f \in R^{p}(X)$. We call such a $g$ an annihilating function. 
The following lemma was proved by Bishop for the sup norm case: We assume that $1<q<2$.

LeMma (2.2). Let $g \in L^{q}(X)$ be an annihilating function. Suppose that $\hat{g}(y)$ is defined and $\neq 0$, and that $(z-y)^{-1} g \in L^{q}(X)$. Then $\hat{g}(y)^{-1}(z-y)^{-1} g$ is a representing function for $y$.

Proof. If $f \in R_{0}(X)$, then $f=f(y)+(z-y) h$ for some $h \in R_{0}(X)$. Hence

$$
\int(z-y)^{-1} f g d m=f(y) \hat{g}(y)+\int h g d m=f(y) \hat{g}(y) .
$$

CoRollary (2.1). Let $g \in L^{q}(X)$ be a representing function for $x$. Let

$$
c(y)=\int(z-x)(z-y)^{-1} g d m=1+(y-x) \hat{g}(y) .
$$

Then $c(y)^{-1}(z-x)(z-y)^{-1} g$ is a representing function for $y$ whenever $c(y)$ is defined and $\neq 0$.

Proof. $(z-x) g$ is an annihilating function.

We now present a lemma of Brennan in $[2$, p. 288] which will be very useful.

Lemma (2.3). If $p>2$, then $R^{p}(X) \neq L^{p}(X)$ if and only if $S^{p}(X)$ has positive 2-dimensional measure.

Proof. Suppose that $S^{p}(X) \neq \varnothing$ and $x \in S^{p}(X)$ is represented by a nonzero function $g \in L^{q}(X)$. Then $R^{p}(X) \neq L^{p}(X)$ because $(z-x) g \in$ $L^{q}(X)$, and $\int(z-x) g f d m=0$ for all $f \in R^{p}(X)$.

Now suppose that $R^{p}(X) \neq L^{p}(X)$ and let $g \in L^{q}(X)$ be a nonzero annihilating function. Then $\hat{g}$ fails to vanish on a set of positive measure in $X$. Hence there is a set $S \subset X$ of positive measure such that for $y \in S, \hat{g}(y) \neq 0$ and $\hat{g}(y)^{-1}(z-y)^{-1} g \in L^{q}(X)$. It follows from Corollary (2.1) that $S \subset$ $S^{p}(X)$, and the lemma is proved.

REMARK. If we know that there is an $x \in S^{2}(X)$, the difficulty in showing that there are other points in $S^{2}(X)$ by the above method is that $z^{-1} \notin L_{\text {loc }}^{2}$

3. Admissible functions. Fix $x \in \mathrm{C}$ and let $\Delta_{n}=\{y \in \mathrm{C}:|y-x|<1 / n\}$. We say that a set $E \subset C$ has full area density at $x$ if $\lim _{n \rightarrow \infty} m(E \cap$ $\left.\Delta_{n}\right) / m\left(\Delta_{n}\right)=1$. Let $F$ be a function defined on $X, x \in X$. We say that $a$ is the approximate limit of $F$ at $x$, and write app $\lim _{y \rightarrow x} F(y)=a$ if there exists a subset $E$ of $X$ having full area density at $x$, such that $\lim _{y \rightarrow x ; y \in E} F(y)=a$. We say that $F$ is approximately continuous at $x$ if app $\lim _{y \rightarrow x} F(y)=F(x)$.

If $\phi$ is a positive function on $(0, \infty)$ with $\lim _{r \rightarrow 0} \phi(r)=0$, we say that $F$ admits $\phi$ as a modulus of approximate continuity at $x$ if $|F(y)-F(x)| \leqslant$ 
$\phi(|y-x|)$ for all $y$ in a set having full area density at $x$. We say that $F$ satisfies an approximate Hölder condition of order $\alpha$ at $x$ if $F$ admits $C r^{\alpha}$ as a modulus of approximate continuity at $x$ for some constant $C$.

Definition (3.1). We say that $\phi$ is an admissible function if

(a) $\phi$ is a positive, nondecreasing function defined on $(0, \infty)$, and

(b) the associated function $\psi$, defined by $\psi(r)=r / \phi(r)$, is nondecreasing, with $\psi(0+)=0$.

EXAMPLE. For any $\alpha, 0 \leqslant \alpha<1, \phi(r)=r^{\alpha}$ is admissible.

REMARKs. 1. If $\phi$ is admissible and $0 \leqslant \beta \leqslant 1$, then $\phi^{\beta}$ is also admissible because $r / \phi^{\beta}(r)=(r / \phi(r)) \cdot \phi^{1-\beta}(r)$.

2. In using an admissible function $\phi$ we will often refer to the triangle inequality: $\phi(r) \leqslant \phi\left(r_{1}\right)+\phi\left(r_{2}\right)$ whenever $r \leqslant r_{1}+r_{2}$. This follows from the definition of an admissible function since

$$
\begin{aligned}
\phi(r) & \leqslant \phi\left(r_{1}+r_{2}\right)=\left(r_{1}+r_{2}\right) / \psi\left(r_{1}+r_{2}\right) \\
& \leqslant r_{1} / \psi\left(r_{1}\right)+r_{2} / \psi\left(r_{2}\right)=\phi\left(r_{1}\right)+\phi\left(r_{2}\right) .
\end{aligned}
$$

Wang introduced a special kind of admissible function in [17, p. 349].

Definition (3.2). We say that the admissible function $\phi$ is nice if $\int_{0}^{1} \phi(r)^{-1} d r<\infty$.

For each $q, 1 \leqslant q<2$, we will be interested in a subset of the set of nice admissible functions.

Definition (3.3). We say that the admissible function $\phi$ is $q$-nice if $\int_{0}^{1} r^{1-q} \phi(r)^{-q} d r<\infty$.

Note that a nice admissible function is 1-nice and that $\phi(r)=r^{\alpha}$ is $q$-nice for $\alpha<(2-q) / q$. When $p>2$, the $q$-nice admissible functions will be the most likely ones to be moduli of approximate continuity for functions in the unit ball of $R^{p}(X)$ at points in $S^{p}(X)$.

The following lemma is due to Wang [17]:

Lemma (3.1). Let $g \in L^{q}(X), q \geqslant 1$, and let $x \in X$. Then there exists a nice admissible function $\phi$ with $\phi(0+)=0$ such that $\phi(|z-x|)^{-1} g \in L^{q}(X)$.

Proof. See Wang [17].

Our proof of the next lemma is in the spirit of Browder's result [3, p. 157]. It will be useful for studying the density of $X$ at points in $S^{p}(X)$. Let $E \subset X$ be measurable. Define $\rho_{n}$ by $\pi \rho_{n}^{2}=m\left(\Delta_{n} \backslash E\right)$. Denote $m \mid \Delta_{n} \backslash E$ by $m_{n}$.

Lemma (3.2). Let $\psi$ be associated with an admissible $\phi$. For $q, 0<q<2$, let $\tau=\psi^{q}$. Then if $g \in L^{1}(X)$,

$$
\lim _{n \rightarrow \infty} \frac{n^{q}}{\rho_{n}^{2-q}} \int \tau(|y-x|) U_{g}^{\tau}(y) d m_{n}(y)=0 .
$$


Proof. Define

$$
F_{n}(\zeta)=n^{q} \rho_{n}^{q-2} \int \psi(|y-x|)^{q} \cdot \psi(|\zeta-y|)^{-q} d m_{n}(y)
$$

Then $F_{n}(x)<\infty$ and if $\zeta \neq x$, we have for large $n$

$$
\left|F_{n}(\zeta)\right| \leqslant n^{q} \rho_{n}^{q} \psi\left(n^{-1}\right)^{q} \cdot \psi\left(|x-\zeta|-n^{-1}\right)^{q} \rightarrow 0 \text { as } n \rightarrow \infty .
$$

Next, we will show that the $F_{n}$ are bounded independently of $n$. Let $D_{n}=$ $\Delta\left(\zeta, \rho_{n}\right)$. Since $\psi^{q}$ is increasing,

$$
\begin{aligned}
\left|F_{n}(\zeta)\right| & \leqslant n^{q} \rho_{n}^{q-2} \psi\left(n^{-1}\right)^{q} \int \psi(|y-\zeta|)^{-q} d m_{n}(y) \\
& \leqslant n^{q} \rho_{n}^{q-2} \psi\left(n^{-1}\right)^{q} \int_{D_{n}} \psi(|y-\zeta|)^{-q} d m(y) \\
& =2 \pi n^{q} \rho_{n}^{q-2} \psi\left(n^{-1}\right)^{q} \int_{0}^{\rho_{n}} \psi(r)^{-q} r d r \\
& \leqslant 2 \pi n^{q} \rho_{n}^{q-2} \psi\left(n^{-1}\right)^{q} \phi\left(\rho_{n}\right)^{q} \int_{0}^{\rho_{n}} r^{1-q} d r \\
& =2 \pi n^{q} \rho_{n}^{q-2} \psi\left(n^{-1}\right)^{q} \phi\left(\rho_{n}\right)^{q} \rho_{n}^{2-q}(2-q)^{-1} \\
& <2 \pi(2-q)^{-1} .
\end{aligned}
$$

Thus, the $F_{n}$ converge boundedly a.e. to zero. We apply the dominated convergence theorem and Fubini's theorem to obtain the lemma.

LEMMA (3.3). Let $\psi$ be associated with an admissible $\phi$. For $0<q<2$, let $\tau=\psi^{q}$. Then if $g \in L^{1}(X)$, and $\delta>0$, the set $E=\left\{y \in \mathbf{C}: \tau(|y-x|) U_{g}^{\tau}(y)\right.$ $<\delta\}$ has full area density at $x$.

Proof. It is sufficient to prove that $\lim _{n \rightarrow \infty} m\left(\Delta_{n} \backslash E\right) / m\left(\Delta_{n}\right)=0$ where $\Delta_{n}=\Delta(x, 1 / n)$. We observe that since

$$
m\left(\Delta_{n} \backslash E\right) \leqslant \delta^{-1} \int_{\Delta_{n}} \tau(|y-x|) U_{g}^{\tau}(y) d m(y),
$$

it is sufficient to prove that

$$
\lim _{n \rightarrow \infty} n^{2} \int_{\Delta_{n}} \tau(|y-x|) U_{g}^{\tau}(y) d m(y)=0
$$

This follows from Lemma (3.2) if we take $E$ in that lemma to be the empty set.

4. The main theorem. The following lemma in the sup norm case is due to Wilken [20]. For $x \in S^{p}(X), p>2$, it gives a condition for $x$ to admit a bounded point derivation of order $s$.

Lemma (4.1). Suppose there exist a representing function $g \in L^{q}(X)$ for 
$x \in S^{p}(X), p>2$, and a nonnegative integer $s$ such that $(z-x)^{-s} g \in$ $L^{q}(X)$. Let $c_{j}=\int(z-x)^{-j} g d m(0 \leqslant j \leqslant s)$ and define $G_{0}, \ldots, G_{s}$ by:

$$
G_{0}=g, \quad G_{j}=(z-x)^{-j} g-\sum_{k<j} c_{j-k} G_{k} .
$$

Then $D_{x}^{j}$ exists, and $D_{x}^{j} f=\int f G_{j} d m$ for all $f \in R^{p}(X), 0 \leqslant j \leqslant s$.

An additional lemma will be needed in proving the theorem.

Lemma (4.2). Let $s$ be a nonnegative integer, and $g \in L^{q}(X), 1 \leqslant q<2$. Suppose that $(z-x)^{-s} g \in L^{q}(X)$. Set $H_{j}=(z-x)^{-j} g(0 \leqslant j \leqslant s)$. For any $f \in L^{p}(X)$ and $y \in \mathbf{C}$

$$
\begin{aligned}
\int(z-y)^{-1} f g d m & \\
= & \sum_{j=1}^{s}(y-x)^{j-1} \int f H_{j} d m+(y-x)^{s} \int(z-y)^{-1} f H_{s} d m .
\end{aligned}
$$

PRoof. Since $H_{j}=(z-x) H_{j+1}$ for $0 \leqslant j \leqslant s$,

$$
\int(z-y)^{-1} f H_{j} d m=\int f H_{j+1} d m+(y-x) \int(z-y)^{-1} f H_{j+1} d m
$$

which implies the lemma.

Our main theorem generalizes the "approximate Taylor's theorem" which Wang obtained for functions in $R(X)$ [17, p. 352].

THEOREM (4.1). Let $\phi$ be an admissible function and $s$ a nonnegative integer. Suppose that $p>2$ and that there is an $x \in S^{p}(X)$ represented by a $g \in$ $L^{q}(X)$ such that $(z-x)^{-s} \phi(|z-x|)^{-1} g \in L^{q}(X)$. Then for every $\varepsilon>0$ there is a set $E$ in $X$ having full area density at $x$ such that for every $f \in R^{p}(X)$

(i) $f=\sum_{j=0}^{s}\left(D_{x}^{j} f\right)(z-x)^{j}+R$ where $R \in R^{p}(X)$ satisfies

(ii) $|R(y)| \leqslant \varepsilon|y-x|^{s} \phi(|y-x|)\|f\|_{p}$ for all $y \in E$, and

(iii) app $\lim _{y \rightarrow x}\left\{R(y) /|y-x|^{s} \phi(|y-x|)\right\}=0$.

Proof. Since $(z-x)^{-s} g \in L^{q}(X)$, Lemma (4.1) implies that the $D_{x}^{j}$ exist for $0 \leqslant j \leqslant s$. To each $D_{x}^{j}, 0 \leqslant j \leqslant s$, there corresponds a constant $C_{j}$ such that $\left|D_{x}^{j} f\right|<C_{j}\|f\|_{p}$ for all $f \in R^{p}(X)$. By Minkowski's inequality there is another constant $C$ such that if $R$ is defined as in (i), $\|R\|_{p} \leqslant C\|f\|_{p}$ for all $f \in R^{p}(X)$.

Choose $\delta>0$ so that $0<C \delta(1-\delta)^{-1}<\varepsilon / 2$. If $y \in E_{1}=\{y \in \mathbf{C}: \mid y-$ $x \mid \tilde{g}(y)<\delta\}$, then $c(y)=1+(y-x) \hat{g}(y)$ is well defined, and $|c(y)| \geqslant 1$ $-\delta$. By Corollary (2.1), 


$$
\begin{aligned}
R(y) & =c(y)^{-1} \int[R(z-x) /(z-y)] g d m \\
& =c(y)^{-1} \int R[1+(y-x) /(z-y)] g d m \\
& =c(y)^{-1}(y-x) \int[R /(z-y)] g d m .
\end{aligned}
$$

Next, we claim that $R(y)=c(y)^{-1}(y-x)^{s+1} \int(z-x)^{-s}(z-y)^{-1} R g d m$. This claim depends on Lemma (4.2). Each of the functions $(z-x)^{-j} g$, $0 \leqslant j \leqslant s$, is a linear combination of functions representing $D_{x}^{k}, 0 \leqslant k \leqslant j$, which implies that $\int(z-x)^{-j} R g d m=0$ for $0 \leqslant j \leqslant s$, and the claim is proved.

Factoring $g=\phi(|z-x|) h$ where $h \in L^{q}(X)$, we obtain by the "triangle inequality" that

$$
|g| \leqslant \phi(|z-y|)|h|+\phi(|y-x|)|h|
$$

Consequently,

$$
\begin{aligned}
|R(y)| \leqslant|c(y)|^{-1}|y-x|^{s+1}[ & \int|z-y|^{-1}|z-x|^{-s} \phi(|z-y|)|R h| d m \\
& \left.+\int|z-y|^{-1}|z-x|^{-s} \phi(|y-x|)|R h| d m\right] .
\end{aligned}
$$

Denote the first integral by $I_{1}$ and the second by $I_{2}$. We have

$$
I_{1}=|c(y)|^{-1}|y-x|^{s} \phi(|y-x|) \psi(|y-x|) \int \psi(|z-y|)^{-1}|z-x|^{-s}|R h| d m .
$$

Let $\tau=\psi^{q}, k=(z-x)^{-s q} h^{q}$, and

$$
E_{2}=\left\{y \in \mathbf{C}: \tau(|y-x|) U_{k}^{\tau}(y)<\delta^{q}\right\}
$$

For $y \in E_{2}$ we apply Hölder's inequality to obtain

$$
\begin{aligned}
I_{1} & \leqslant(1-\delta)^{-1}|y-x|^{s} \phi(|y-x|) \tau(|y-x|)^{1 / q}\left\{\int|R|^{p} d m\right\}^{1 / p}\left\{U_{k}^{\tau}(y)\right\}^{1 / q} \\
& \leqslant(1-\delta)^{-1}|y-x|^{s} \phi(|y-x|) C\|f\|_{p} \delta \\
& \leqslant(\varepsilon / 2)|y-x|^{s} \phi(|y-x|)\|f\|_{p} .
\end{aligned}
$$

To estimate $I_{2}$ we define

$$
E_{3}=\left\{y \in \mathrm{C}:|y-x|^{q} U_{k}^{q}(y)<\delta^{q}\right\} \quad \text { and let } y \in E_{2} \cap E_{3} .
$$

By Hölder's inequality, 


$$
\begin{aligned}
I_{2} & \leqslant(1-\delta)^{-1}|y-x|^{s} \phi(|y-x|)|y-x| \int|z-y|^{-1}|z-x|^{-s}|R h| d m \\
& \leqslant(1-\delta)^{-1}|y-x|^{s} \phi(|y-x|)|y-x|\left\{\int|R|^{p} d m\right\}^{1 / p}\left\{U_{k}^{q}(y)\right\}^{1 / q} \\
& \leqslant(1-\delta)^{-1}|y-x|^{s} \phi(|y-x|) C\|f\|_{p} \delta \\
& \leqslant(\varepsilon / 2)|y-x|^{s} \phi(|y-x|)\|f\|_{p} .
\end{aligned}
$$

By Lemma (3.3) the set $E=E_{2} \cap E_{3}$ has full area density at $x$, and we have proved that for $y \in E$

$$
|R(y)| \leqslant I_{1}+I_{2} \leqslant \varepsilon|y-x|^{s} \phi(|y-x|)\|f\|_{p}
$$

for any $f \in R^{p}(X)$. To prove (iii) let $L_{y} f=R(y) /|y-x|^{s} \phi(|y-x|)$. The above result implies that $\left\|L_{y}\right\| \leqslant \varepsilon$ for $y \in E$. Let $y \rightarrow x$ in such a way that $y$ stays in $E$. Then $I_{y} f \rightarrow 0$ as $y \rightarrow x$ for $f \in R_{0}(X)$, and since $R_{0}(X)$ is dense in $R^{p}(X)$, (iii) follows.

An interesting consequence of the above theorem is that we can take the limit of Newton quotients in the set $E$ to evaluate $D_{x}^{1} f$. For $f$ a function defined on a subset of $X, h \in \mathbf{C}$, we set

$$
\Delta_{h} f=f(z+h)-f
$$

so $\Delta_{h} f$ is a function defined on a subset of $X$. We define inductively $\Delta_{h}^{0}=\mathrm{id}$, $\Delta_{h}^{j}=\Delta_{h} \circ \Delta^{j-1}$ for $j \geqslant 1$. The sup norm version of the following corollary is proved in [17].

COROLlaRY (4.1). If $x$ admits a bounded point derivation of order $s$ on $R^{p}(X), p>2$, then for all $f \in R^{p}(X)$

$$
D_{x}^{s} f=\underset{h \rightarrow 0}{\operatorname{app} \lim } \frac{\Delta_{h}^{s} f(x)}{s ! h^{s}} .
$$

LeMma (4.3). Let $\phi$ be a q-nice admissible function. If $x \in S^{p}(X), p>2$, then $\left\{y \in X: \exists\right.$ a function $g_{y}$ that represents $y$ for $R^{p}(X)$ and satisfies $\left.\phi(|z-y|)^{-1} g_{y} \in L^{q}(X)\right\}$ has full area density at $x$.

Proof. Let $g \in L^{q}(X)$ represent $x$.

Let

$$
F=\left\{y \in \mathbf{C}: \int|z-y|^{-q} \phi(|z-y|)^{-q}|g|^{q} d m<\infty\right\} .
$$

Since $|z|^{-q} \phi(|z|)^{-q}$ is locally summable with respect to $m, m(\mathbf{C} \backslash F)=0$. Fix $\delta, 0<\delta<1$, and put $E=F \cap E_{1}$ where $E_{1}=\{y \in \mathrm{C}:|y-x| \tilde{g}(y)<\delta\}$. By Lemma (3.3) the set $E$ has full area density at $x$. For each $y \in E$ the function $g_{y}=c(y)^{-1}[(z-x) /(z-y)] g$ represents $y$. Moreover, 


$$
\begin{aligned}
\int \phi(|z-y|)^{-q}\left|g_{y}\right|^{q} d m & =|c(y)|^{-q} \int|z-y|^{-q} \phi(|z-y|)^{-q}|z-x|^{q}|g|^{q} d m \\
& \leqslant C \int|z-y|^{-q} \phi(|z-y|)^{-q}|g|^{q} d m<\infty .
\end{aligned}
$$

This proves the lemma.

COROllary (4.2). Suppose that $\phi$ is $q$-nice. Then at almost every point of $S^{p}(X), p>2$, the functions in the unit ball of $R^{p}(X)$ admit $\phi$ as a modulus of approximate continuity.

Proof. Combine Theorem (4.1) with Lemma (4.3).

In particular, it follows that at a.e. $x \in S^{p}(X), p>2$, the unit ball of $R^{p}(X)$ satisfies an approximate uniform Hölder condition of order $\alpha$ for every $\alpha<(2-q) / q$.

LeMmA (4.4). Let $\phi$ be admissible and $g \in L^{q}(X), 1 \leqslant q<2$. Then if $\phi(|z-x|)^{-1} g \in L^{q}(X), \delta>0$, and

$$
E=\left\{y \in \mathbf{C}:|y-x|^{q} \int|y-z|^{-q}|g|^{q} d m<\delta\right\},
$$

it follows that $m\left(\Delta_{n} \backslash E\right)=o\left(\phi\left(n^{-1}\right)^{2} / n^{2}\right)$.

Proof. We observe that

$$
m\left(\Delta_{n} \backslash E\right) \leqslant \delta^{-1} \int|y-x|^{q} \int|z-y|^{-q}|g|^{q} d m d m_{n}(y) .
$$

Factor $g=\phi(|z-x|) h$ where $h \in L^{q}(X)$. Then

$$
|g|^{q} \leqslant C\left[\phi(|z-y|)^{q}|h|^{q}+\phi(|y-x|)^{q}|h|^{q}\right]
$$

where $C$ is some constant. We have

$$
\begin{aligned}
& m\left(\Delta_{n} \backslash E\right) \leqslant \delta^{-1} C\left[\int|y-x|^{q} \int|z-y|^{-q} \phi(|z-y|)^{q}|h|^{q} d m d m_{n}(y)\right. \\
&\left.+\int|y-x|^{q} \int|z-y|^{-q} \phi(|y-x|)^{q}|h|^{q} d m d m_{n}(y)\right] .
\end{aligned}
$$

By substituting $|y-x|^{q}=\phi(|y-x|)^{q} \psi(|y-x|)^{q}$ in the first integral, and using the fact that $\phi(|y-x|)^{q} \leqslant \phi\left(n^{-1}\right)^{q}$ for $y \in \Delta_{n}$, we obtain

$$
\begin{aligned}
m\left(\Delta_{n} \backslash E\right) \leqslant \delta^{-1} C \phi\left(n^{-1}\right)^{q}[\psi(\mid y & -x \mid)^{q} \int \psi(|z-y|)^{-q}|h|^{q} d m d m_{n}(y) \\
& \left.+\int|y-x|^{q} \int|z-y|^{-q}|h| d m d m_{n}(y)\right] .
\end{aligned}
$$

Let $A_{n}$ denote the sum of the two integrals on the right. Replacing $m\left(\Delta_{n} \backslash E\right)$ by $\pi \rho_{n}^{2}$, we obtain

$$
\pi \rho_{n}^{2} \leqslant \delta^{-1} C \phi\left(n^{-1}\right)^{q} \rho_{n}^{2-q_{n}-q}\left(A_{n}\right)
$$


where $\lim _{n \rightarrow \infty} A_{n}=0$ by Lemma (3.2). Divide both sides by $\rho_{n}^{2-q}$ to get

$$
\pi \rho_{n}^{q} \leqslant \delta^{-1} C \phi\left(n^{-1}\right)^{q} n^{-q}\left(A_{n}\right) .
$$

Now raise both sides to the power $2 / q$, and the conclusion of the lemma follows.

In the next corollary we consider functions $f \in R^{p}(X)$ to be defined on $\mathbf{C}$ by setting $f(x)=0$ for $x \notin X$.

Corollary (4.3). Let $\varepsilon>0$. If $x \in S^{p}(X), p>2$, is represented by $g \in L^{q}(X)$, and $(z-x)^{-\alpha} g \in L^{q}(X)$ for some $\alpha>q-1$, then there is an integer $N_{x}$ depending on $x$ such that for $n>N_{x}$

$$
m\left(\Delta_{n}\right)^{-1} \int_{\Delta_{n}}|f-f(x)| d m \leqslant \varepsilon\|f\|_{p} \text { for all } f \in R^{p}(X) .
$$

Proof. Let $E$ be the set in the conclusion of Theorem (4.1) when $\varepsilon / 2$ and $x \in S^{p}(X)$ are given and $\phi(r) \equiv 1$.

$$
\begin{aligned}
m\left(\Delta_{n}\right)^{-1} & \int_{\Delta_{n}}|f-f(x)| d m \\
& \leqslant m\left(\Delta_{n}\right)^{-1}\left[\int_{\Delta_{n} \cap E}|f-f(x)| d m+\int_{\Delta_{n} \backslash E}|f-f(x)| d m\right] \\
& \leqslant(\varepsilon / 2)\|f\|_{p} m\left(\Delta_{n}\right)^{-1} m\left(\Delta_{n} \cap E\right)+\pi^{-1} n^{2} \int_{\Delta_{n} \backslash E}|f-f(x)| d m \\
& \leqslant(\varepsilon / 2)\|f\|_{p}+\pi^{-1} n^{2} \int_{\Delta_{n} \backslash E}|f-f(x)| d m .
\end{aligned}
$$

Let $\chi_{\Delta_{n} \backslash E}$ be the characteristic function of $\Delta_{n} \backslash E$. Then by Hölder's inequality,

$$
\begin{aligned}
\pi^{-1} n^{2} \int_{\Delta_{n} \backslash E}|f-f(x)| d m & =\pi^{-1} n^{2} \int \chi_{\Delta_{n} \backslash E}|f-f(x)| d m \\
& \leqslant C n^{2}\left[m\left(\Delta_{n} \backslash E\right)\right]^{1 / q}\|f\|_{L^{P}\left(\Delta_{n} \backslash E\right)}
\end{aligned}
$$

where $C$ is a constant. By Lemma (4.4)

$$
\left[m\left(\Delta_{n} \backslash E\right)\right]^{1 / q}=o\left(n^{-(2 / q)-(2 \alpha / q)}\right) .
$$

Thus if $\alpha>q-1$, we can choose an integer $N_{x}$ so that $n>N_{x}$ implies that $C n^{2}\left[m\left(\Delta_{n} \backslash E\right)\right]^{1 / q} \leqslant \varepsilon / 2$. Hence,

$$
\begin{aligned}
m\left(\Delta_{n}\right)^{-1} \int_{\Delta_{n}}|f-f(x)| d m & \leqslant(\varepsilon / 2)\|f\|_{p}+(\varepsilon / 2)\|f\|_{L^{p}\left(\Delta_{n} \backslash E\right)} \\
& \leqslant \varepsilon\|f\|_{p^{*}}
\end{aligned}
$$

This completes the proof. 
COROLLARY (4.4). If $p>2+\sqrt{2}$, then for a.e. $x \in S^{p}(X)$,

$$
\lim _{n \rightarrow \infty} m\left(\Delta_{n}\right)^{-1} \int_{\Delta_{n}}|f-f(x)| d m=0 \text { for any } f \in R^{p}(X) .
$$

Proof. This follows from Lemma (4.3) and Corollary (4.3).

Given $f \in L^{1}(d m)$, the set of points $x \in \mathbf{C}$ such that

$$
\lim _{n \rightarrow \infty} m\left(\Delta_{n}\right)^{-1} \int_{\Delta_{n}}|f-f(x)| d m=0
$$

is called the Lebesgue set of $f$. For an arbitrary $f \in L^{1}(d m)$, a.e. $(m)$ point $x \in \mathbf{C}$ belongs to the Lebesgue set of $f$ (see [5, p. 156]). The above corollary identifies points belonging to the Lebesgue sets of all $f \in R^{p}(X)$. It would be interesting to know whether the corollary holds for $p>2$.

\section{Part II. Capacity and bounded point eValuations}

1. Capacity theorems. Before proving a capacity result about bounded point evaluations, we will need two lemmas of Hedberg [9]. Let $\Omega$ denote the complex plane when $p>2$ and the unit disk when $p=2$.

Definition (1.1). Let $X \subset \Omega$ be a compact set. Then

$$
\Gamma_{q}(X)=\inf _{\omega} \int|\operatorname{grad} \omega|^{q} d m
$$

where the inf is taken over Lipschitz functions $\omega$ with compact support contained in $\Omega$ such that $\omega(z) \geqslant 1$ on $X$.

For noncompact sets $F, q$-capacity is defined by $\Gamma_{q}(F)=\sup _{K \subset F} \Gamma_{q}(K), K$ compact.

Let $U$ be an open set (bounded if $p=2$ ) in the complex plane and denote by $L_{a}^{p}(U)$ the space of analytic functions in $L^{p}(U)$. If $f$ is analytic in $\Omega \backslash X$ where $X \subset \Omega$ is compact, we write $\alpha(f)=(2 \pi i)^{-1} \int_{C} f(z) d z$ where $C$ is any Jordan curve in $\Omega$ enclosing $X$.

Lemma (1.1). Let $X \subset \Omega$ be compact. Then there are positive constants $C_{1}$ and $C_{2}$, depending only on $p$, such that

$$
C_{1} \Gamma_{q}(X)^{1 / q} \leqslant \sup _{f}|\alpha(f)| \leqslant C_{2} \Gamma_{q}(X)^{1 / q}
$$

where the sup is taken over functions $f$ in $L_{a}^{p}(\Omega), 2 \leqslant p<\infty$, with $\int_{\Omega \backslash X}|f(z)|^{p} d m \leqslant 1$.

We denote the annulus $\left\{z: 2^{-n-1} \leqslant|z-x| \leqslant 2^{-n}\right\}$ by $A_{n}(X)$. We write $A_{n}=A_{n}(0)$.

Lemma (1.2). Let $X \subset \Omega$ be compact. There is a constant $C$, depending only on $p$, such that for $z \notin A_{n-1} \cup A_{n} \cup A_{n+1}$ 


$$
|f(z)| \leqslant \frac{C \Gamma_{q}\left(A_{n} \backslash X\right)^{1 / q}}{|| z\left|-2^{-n}\right|}\|f\|_{\Omega \backslash X, p}
$$

for $f$ analytic outside $A_{n} \backslash X, f(\infty)=0$ and $\int_{\Omega \backslash X}|f(z)|^{p} d m<\infty$.

The following theorem was proved in the sup norm case by Wang [18, p. 223]. Wang essentially followed O'Farrell [13], who elaborated on a method of Gamelin [7, p. 206]. We assume that $x=0$ and that $0 \in \partial X$.

THEOREM (1.1). Let $\phi$ be an admissible function and $s$ a nonnegative integer. Suppose that there is a function $v \in L^{q}(X)$ which represents 0 for $R^{p}(X)$ such that $|z|^{-s} \phi(|z|)^{-1} v \in L^{q}(X)$. Then

$$
\sum_{1}^{\infty} 2^{q(s+1) n} \phi\left(2^{-n}\right)^{-q} \Gamma_{q}\left(A_{n} \backslash X\right)<\infty .
$$

Proof. Suppose that

$$
\sum_{1}^{\infty} 2^{q(s+1) n} \phi\left(2^{-n}\right)^{-q} \Gamma_{q}\left(A_{n} \backslash X\right)=\infty .
$$

We will show that this leads to a contradiction. We may assume that for each $n$

$$
2^{q(s+1) n} \pi\left(2^{-n}\right)^{-q} \Gamma_{q}\left(A_{n} \backslash X\right) \leqslant 1 .
$$

If not, choose $Y_{n}$ compact, $Y_{n} \subset A_{n}$ such that

$$
\frac{1}{2} \leqslant 2^{q(s+1) n} \phi\left(2^{-n}\right)^{-q} \Gamma_{q}\left(A_{n} \backslash X \cup Y_{n}\right) \leqslant 1,
$$

and set $Y=\overline{\cup Y}_{n} \cup X$. Then define $v^{*}(z)=v(z)$ for $z \in X$ and $v^{*}(z)=0$ for $z \in Y \backslash X$. Clearly, $|z|^{-s} \phi(|z|)^{-1} v^{*} \in L^{q}(Y)$ and $v^{*}$ represents 0 for $R^{P}(Y)$.

Now choose integers $M_{1} \leqslant N_{1}<M_{2} \leqslant N_{2}<\cdots$ so that

$$
1 \leqslant \sum_{n=M_{j}}^{N_{j}} 2^{q(s+1) n} \phi\left(2^{-n}\right)^{-q} \Gamma_{q}\left(A_{n} \backslash X\right) \leqslant 2 .
$$

For each $n$ we choose by Lemma (1.1) compact sets $K_{n} \subset A_{n} \backslash X$ and functions $f_{n} \in L_{a}^{p}\left(\Omega \backslash K_{n}\right)$ so that:

$$
\begin{gathered}
\left|\alpha\left(f_{n}\right)\right| \geqslant C_{1} 2^{-1} \Gamma_{q}\left(A_{n} \backslash X\right)^{1 / q}\left\{\int_{\Omega \backslash K_{n}}\left|f_{n}(z)\right|^{p} d m\right\}^{1 / p} \\
=C_{1} 2^{-1} \Gamma_{q}\left(A_{n} \backslash X\right)^{1 / q}\left\|f_{n}\right\|_{\Omega \backslash K_{n}, p} \\
f_{n}=0 \quad \text { on } K_{n} \text { and } \\
\left\|f_{n}\right\|_{\Omega, p}=2^{q(s+1) n} \phi\left(2^{-n}\right)^{-q} \Gamma_{q}\left(A_{n} \backslash X\right)^{1 / p}
\end{gathered}
$$


Let $g_{j}(z)=\phi(|z|) z^{s+1} \sum_{n=M_{j}}^{N_{j}} f_{n}(z)$. We will show that $\left\|g_{j}\right\|_{X_{p}} \leqslant C$ for all $j$. In the following discussion $C$ will denote any constant that is independent of $n$ and $j$. Lemma (II.1.2) implies that for $z \in A_{k}, k<n-1$,

$$
\left|f_{n}(z)\right| \leqslant C 2^{q(s+1) n+k} \phi\left(2^{-n}\right)^{-q} \Gamma_{q}\left(A_{n} \backslash X\right)
$$

and for $z \in A_{k}, k>n+1$,

$$
\left|f_{n}(z)\right| \leqslant C 2^{q(s+1) n+n} \phi\left(2^{-n}\right)^{-q} \Gamma_{q}\left(A_{n} \backslash X\right) .
$$

We may assume that $X \subset\{|z| \leqslant 1\}$. Then for $z \in A_{k} \cap X, k<n-1$,

$$
\phi(|z|)|z|^{s+1}\left|f_{n}(z)\right| \leqslant C 2^{q(s+1) n} \phi\left(2^{-n}\right)^{-q} \Gamma_{q}\left(A_{n} \backslash X\right) .
$$

For $z \in A_{k}, k>n+1$,

$$
\begin{aligned}
\phi(|z|)|z|^{s+1}\left|f_{n}(z)\right| & \leqslant C 2^{q(s+1) n+n-(s+1) n} \phi\left(2^{-n}\right)^{1-q} \Gamma_{q}\left(A_{n} \backslash X\right) \\
& \leqslant C 2^{q(s+1) n} \phi\left(2^{-n}\right) \Gamma_{q}\left(A_{n} \backslash X\right) .
\end{aligned}
$$

Now

$$
\begin{aligned}
\int_{X}\left|g_{j}(z)\right|^{p} d m & =\sum_{k=0}^{\infty} \int_{A_{k} \cap X}\left|\sum_{n=M_{j}}^{N_{j}} \phi(|z|) z^{s+1} f_{n}(z)\right|^{p} d m \\
& <C \sum_{k=0}^{\infty} \int_{A_{k} \cap X}\left\{\left[\left.\left|\sum_{n=M_{j} ; n \neq k-1, k, k+1} \phi(|z|)\right| z\right|^{s+1}\left|f_{n}(z)\right|\right]^{p}\right. \\
& \left.+\sum_{n=k-1}^{k+1}\left(\phi(|z|)|z|^{s+1}\left|f_{n}(z)\right|\right)^{p}\right\} d m .
\end{aligned}
$$

By the above estimates and the choice of $M_{j}, N_{j}$, we have for $z \in A_{k}$

$$
\sum_{n=\max \left(k+2, M_{j}\right)}^{N_{j}} \phi(|z|)|z|^{s+1}\left|f_{n}(z)\right| \leqslant C \sum_{n=M_{j}}^{N_{j}} 2^{q(s+1) n} \phi\left(2^{-n}\right)^{-q} \Gamma_{q}\left(A_{n} \backslash X\right) \leqslant C .
$$

Similarly,

$$
\sum_{n=M_{j}}^{\min \left(k-2, N_{j}\right)} \phi(|z|)|z|^{s+1}\left|f_{n}(z)\right| \leqslant C \sum_{n=M_{j}}^{N_{j}} 2^{q(s+1) n} \phi\left(2^{-n}\right)^{-q} \Gamma_{q}\left(A_{n} \backslash X\right) \leqslant C .
$$

Thus

$$
\sum_{k=0}^{\infty} \int_{A_{k} \cap X}\left[\sum_{n=M_{j} ; n \neq k-1, k, k+1}^{N_{j}} \phi(|z|)|z|^{s+1}\left|f_{n}(z)\right|\right)^{p} d m<C .
$$

Next, we estimate 


$$
\sum_{k=0}^{\infty} \int_{A_{k} \cap X_{n=k-1}} \sum_{n=1}^{k+1}\left(\phi(|z|)|z|^{s+1}\left|f_{n}(z)\right|\right)^{p} d m .
$$

For each $k$,

$$
\begin{aligned}
\int_{A_{k} \cap X}\left(\phi(|z|)|z|^{s+1}\left|f_{k-1}(z)\right|\right)^{p} d m \\
\quad \leqslant C\left(\phi\left(2^{-k+1}\right)^{p} 2^{-p(k-1)}\left\|f_{k-1}\right\|_{X, p}^{p}\right) \\
\quad \leqslant C \phi\left(2^{-k+1}\right)^{p-p q} 2^{(k-1)[-p+p q(s+1)]} \Gamma_{q}\left(A_{k-1} \backslash X\right) \\
\leqslant C 2^{q(s+1)(k-1)} \phi\left(2^{-k+1}\right)^{-q} \Gamma_{q}\left(A_{k-1} \backslash X\right)
\end{aligned}
$$

and similarly for $f_{k}$ and $f_{k+1}$. Thus

$$
\begin{gathered}
\sum_{k=0}^{\infty} \int_{A_{k} \cap X_{n}} \sum_{k-1}^{k+1}\left(\phi(|z|)|z|^{s+1}\left|f_{n}(z)\right|\right)^{p} d m \\
\leqslant C \sum_{k=M_{j}}^{N_{j}} 2^{q(s+1) k} \phi\left(2^{-k}\right)^{-q} \Gamma_{q}\left(A_{k} \backslash X\right) \\
\leqslant C \text { by choice of } M_{j} \text { and } N_{j} .
\end{gathered}
$$

Combining the above estimates, we obtain

$$
\int_{X}\left|g_{j}\right|^{p} d m \leqslant C \text { for all } j .
$$

Next we pass to a subsequence of the $\left\{g_{j}\right\}$ that converges weakly to $g \in L^{p}(X)$. Denote the subsequence also by $\left\{g_{j}\right\}$. We form $h_{j}(z)=$ $z \phi(|z|)^{-1} g_{j}(z)$ and $F_{j}(z)=z^{-s-1} h_{j}(z)$, which are analytic in $\mathrm{C} \backslash \Delta\left(0,2^{-M_{j}}\right)$. By the above estimates the functions $h_{j}$ and $F_{j}$ are uniformly bounded on compact subsets of $\mathbf{C} \backslash\{0\}$. Hence, there are subsequences that converge uniformly on compact subsets of $\mathbf{C} \backslash\{0\}$ to $h(z)=z \phi(|z|)^{-1} g(z)$ and $F(z)=$ $z^{-s-1} h(z)$ respectively.

We claim that $h$ is a polynomial of degree $s+1$ with $h(0)=0$. The above estimates show that there is a number $M>0$ that bounds the $h_{j}$ in the following sense: to any $z \in \Delta(0,1) \backslash\{0\}$ there corresponds an integer $J$ such that for $j>J$ and $|\zeta| \geqslant|z|,\left|h_{j}(\zeta)\right|<M$. This implies that $h$ is bounded near 0 , so $h$ is entire and $\lim _{z \rightarrow 0} h(z)=0$. To show that $h$ is a polynomial we consider

$$
\lim _{z \rightarrow \infty} z^{-s-1} h(z)=F(\infty)=\lim _{j \rightarrow \infty} F_{j}(\infty) .
$$

For all $j, F_{j}(\infty)=\sum_{n=M_{j}}^{N_{j}} f_{n}(\infty)$ lies in $\left[C_{1} / 2,3 C_{2}\right]$ where $C_{1}$ and $C_{2}$ are the constants of Lemma (1.1). Therefore, we have that $\lim _{j \rightarrow \infty} F_{j}(\infty)=\beta \in\left[C_{1}\right.$, $\left.2 C_{2}\right]$, and 


$$
h(z)=\beta z^{s+1}+\sum_{1}^{s} \beta_{i} z^{i} \text { where } \beta_{i} \text { is a constant for each } i .
$$

Thus

$$
g_{j}=\phi(|z|) z^{-1} h_{j} \rightarrow \phi(|z|) z^{-1} h=\beta \phi(|z|) z^{s}+\sum_{1}^{s} \beta_{i} \phi(|z|) z^{i-1}
$$

weakly and pointwise on each bounded subset of $\mathbf{C} \backslash\{0\}$.

This means that if $u \in L^{q}(X)$, then

$$
\int g_{j} u d m \rightarrow \int \beta \phi(|z|) z^{s} u d m+\sum_{1}^{s} \beta_{i} \int \phi(|z|) z^{i-1} u d m .
$$

Wilkin's lemma (Lemma (I.4.1)) and the original hypothesis imply that there is a function $v_{s} \in L^{q}(X)$ which is a linear combination of the functions $z^{-j} v$, $0 \leqslant j \leqslant s$, such that

$$
\int f v_{s} d m=\frac{f^{(s)}(0)}{s !}
$$

for all $f \in R_{0}(X)$. Taking $u=\phi(|z|)^{-1} v_{s}$, we get a contradiction.

The next theorem may be proved in a similar wày, and we omit many of the details.

THEOREM (1.2). Let $\phi$ be an admissible function and $s$ a nonnegative integer. Suppose that there is a function $v \in L^{q}(X)$ representing 0 for $R^{p}(X)$ such that $|z|^{-s} \phi(|z|)^{-1} v \in L^{q}(X)$. Then

$$
\lim _{r \rightarrow 0} r^{-q s-q} \phi(r)^{-q} \Gamma_{q}(\Delta(0, r) \backslash X)=0 .
$$

PRoof. Suppose that there is a sequence $r_{n} \rightarrow 0$ and a $b>0$ such that

$$
r_{n}^{-q s-q} \phi\left(r_{n}\right)^{-q} \Gamma_{q}\left(\Delta\left(0, r_{n}\right) \backslash X\right)>b \text { for all } r_{n} .
$$

We may assume as before that

$$
2^{q(s+1) n} \phi\left(2^{-n}\right)^{-q} \Gamma_{q}\left(A_{n} \backslash X\right) \leqslant 1 \text { for all } n .
$$

Note that if $2^{-k}>r_{n}$, and $\left|2^{-k}-r_{n}\right|<2^{-k-1}$,

$$
2^{q(s+1)} \sum_{n=k}^{\infty} 2^{q(s+1) n} \phi\left(2^{-n}\right)^{-q} \Gamma_{q}\left(A_{n} \backslash X\right)>b .
$$

Thus there is a sequence of integers $M_{1} \leqslant N_{1}<M_{2} \leqslant N_{2}<\cdots$ such that

$$
2>\sum_{n=M_{j}}^{N_{j}} 2^{q(s+1) n} \phi\left(2^{-n}\right)^{-q} \Gamma_{q}\left(A_{n} \backslash X\right)>2^{-q(s+1)} b
$$

for all $j$. The proof then proceeds as before.

2. Density at bounded point evaluations. We will get an estimate for $\Gamma_{q}$ capacity in terms of the measure $m$. The following lemma is in [4]. 
LEMMA (2.1). Let $\mu$ be a measure of total mass 1 (i.e. $\int d \mu=1$ ). If $1<q<2$ and $p=q /(q-1)$, then

$$
\int_{\mathbf{C}}\left\{\int|\zeta-z|^{-1} d \mu(\zeta)\right\}^{p} d m \leqslant C\left\{\sup _{z \in \mathbf{C}} \int|\zeta-z|^{q-2} d \mu(\zeta)\right\}^{p-1}
$$

where $C$ is some constant depending only on $p$.

LEMMA (2.2). For each $q, 1<q<2$, there is a positive constant $C$ such that

$$
\Gamma_{q}(X) \geqslant C m(X)^{(2-q) / 2}
$$

for all compact sets $X \subset \mathbf{C}$.

Proof. Define $f=m(X)^{-1} \int_{X}(z-\zeta)^{-1} d m(\zeta)$. Then $f$ is analytic in $\mathbf{C} \backslash X$ and $f^{\prime}(\infty)=1$. To estimate $\|f\|_{p, \mathbf{C} \backslash X}$ we apply Lemma (II.2.1) with $\mu=$ $m(X)^{-1} \chi_{X}$ where $\chi_{X}$ is the characteristic function of $X$. We get

$$
\|f\|_{\mathbf{C} \backslash X, p} \leqslant C\left\{\sup _{z \in \mathbf{C}} m(X)^{-1} \int_{X}|z-\zeta|^{q-2} d m(\zeta)\right\}^{1 / q}
$$

We will use $C$ to denote any constant depending only on $p$. Choose $R>0$ so that $R^{2}=m(X)$, and let $D=\Delta(\zeta, R)$. Then since $r^{q-2}$ is a decreasing function of $r$,

$$
\begin{aligned}
m(X)^{-1} \int_{X}|z-\zeta|^{q-2} d m(\zeta) & \leqslant \pi^{-1} R^{-2} \int_{D}|z-\zeta|^{q-2} d m(\zeta) \\
& =\pi^{-1} R^{-2} \int_{0}^{2 \pi} \int_{0}^{R} r^{q-2} r d r d \theta \\
& =2 R^{-2} \int_{0}^{R} r^{q-1} d r \\
& =2(q-1)^{-1} R^{-2} R^{q}=2(q-1)^{-1} R^{q-2}
\end{aligned}
$$

Applying the above inequality for $\|f\|_{\mathbf{C} \backslash X, p}$, we have

$$
\|f\|_{\mathbf{C} \backslash X, p} \leqslant C R^{(q-2) / q} \text {. }
$$

Define $g=f /\|f\|_{\mathbf{C} \backslash X, p}$. Then $g$ is analytic in $\mathbf{C} \backslash X$ and $\|g\|_{\mathbf{C} \backslash X, p}=1$. Moreover,

$$
g^{\prime}(\infty)=f^{\prime}(\infty) /\|f\|_{\mathrm{C} \backslash X, p} \geqslant C R^{(2-q) / q} \geqslant C m(X)^{(2-q) / 2 q} .
$$

By Lemma (II.1.1) we conclude that

$$
\Gamma_{q}(X) \geqslant C m(X)^{(2-q) / 2}
$$

and the proof is complete.

COROLlaRY (2.1). Let $\phi$ be an admissible function and $s$ a nonnegative integer. Suppose that there is a function $v \in L^{q}(X)$ representing 0 for $R^{p}(X)$, 
$p>2$, such that $|z|^{-s} \phi(|z|)^{-1} v \in L^{q}(X)$. Then

$$
m\left(\Delta\left(0, n^{-1}\right) \backslash X\right)=o\left(\phi\left(n^{-1}\right)^{2 t}\left(n^{-1}\right)^{2 t(s+1)}\right), \text { where } t=q /(2-q) .
$$

Proof. This follows from Theorem (II.1.2) and Lemma (II.2.2).

3. An example. In this section we use Hedberg's capacity theorems to construct a Swiss cheese $Y$ such that $\cap_{p>2}^{\infty} S^{p}(Y)=\{0\}$. Let $X_{0}$ be the closure of a set having positive measure whose boundary consists of finitely many analytic curves. The first step is to show that for a given $\varepsilon>0$ and $p>2$ one can construct a Swiss cheese $X=X_{0} \backslash \cup_{i=1}^{\infty} D_{i}$ such that:

(1) $\sum_{i=1}^{\infty} r_{i}^{2-q}<\varepsilon$ where $r_{i}$ is the radius of $D_{i}$; and

(2) for some $p^{\prime}, p>p^{\prime}>2, S^{p^{\prime}}(X)=\varnothing$. For $n=1,2, \ldots$ we define $X_{n}$ inductively by letting $X_{n}=X_{n-1} \backslash G_{n}$ where $G_{n}=U\left\{\Delta\left(t 2^{-n},\left(\varepsilon 2^{-n}\right)^{3 /(2-q)}\right)\right.$, where the summation is taken over all Gaussian integers $t$ such that $\left|t 2^{-n}\right| \leqslant$ 1). Then set $X=\bigcap_{n=0}^{\infty} X_{n}$. Since each $G_{n}$ consists of $\leqslant 2^{2 n}$ disks

$$
\sum_{i=1}^{\infty} r_{i}^{2-q}<\sum_{i=1}^{\infty} 2^{2 i}\left[\left(\varepsilon 2^{-i}\right)^{3 /(2-q)}\right]^{2-q}=\varepsilon
$$

Now choose $q^{\prime}, q<q^{\prime}<2$, so that $3\left(2-q^{\prime}\right) /(2-q)<q^{\prime}$. Let $x \in X$. We claim that $x \notin S^{p^{\prime}}(X)$ where $1 / p^{\prime}+1 / q^{\prime}=1$. Within any disk centered at $x$ and having radius $2^{-n}$, there is a disk in $\mathbf{C} \backslash X$ having radius at least $4^{-1}\left(\varepsilon 2^{-r}\right)^{3 /(2-q)}$. Hence

$$
\begin{aligned}
\lim _{n \rightarrow \infty} 2^{n q^{\prime}} \Gamma_{q^{\prime}} & \left(\Delta\left(x, 2^{-n}\right) \backslash X\right) \\
& \geqslant 4^{q^{\prime}-2} \cdot \lim _{n \rightarrow \infty} 2^{n q^{\prime}}\left(\varepsilon 2^{-n}\right)^{3\left(2-q^{\prime}\right) /(2-q)}>0 .
\end{aligned}
$$

Thus by Theorem (II.1.2), $x \notin S^{q^{\prime}}(X)$, and $X$ is the desired set.

Given $\varepsilon_{j} \downarrow 0$ and $p_{j} \downarrow 2$, it is possible by the above construction to remove open disks $D_{j k}$ of radius $r_{j k}$ from $A_{j}(0)$ to obtain a Swiss cheese $Y_{j}^{*}$ such that $\sum_{k=1}^{\infty} r_{j k}^{2-q_{j}}<\varepsilon_{j}\left(1 / p_{j}+1 / q_{j}=1\right)$, and $S^{p_{j}^{\prime}}\left(Y_{j}\right)=\varnothing$ for some $p_{j}^{\prime}, p_{j}>p_{j}^{\prime}>2$. Choose the $\varepsilon_{j}$ so that $\sum_{j=1}^{\infty} 2^{2 j} \varepsilon_{j}<\infty$, and define $Y=\cup_{j=0}^{\infty} Y_{j} \cup\{0\}$.

We will use Hedberg's theorem [9] to prove that for any $p>2,0 \in S^{p}(Y)$. Let $p>2$. There is an integer $J$ such that $p>p_{j}>2$ for $j \geqslant J$. Hence,

$$
\sum_{j=J}^{\infty} 2^{j q} \Gamma_{q}\left(A_{j}(0) \backslash X\right)<C \sum_{j=J}^{\infty} 2^{j q} \sum_{k=1}^{\infty} r_{j k}^{2-q}<C \sum_{j=J}^{\infty} 2^{j q} \varepsilon_{j}<\infty .
$$

By Hedberg's theorem $0 \in S^{p}(Y)$, and since $p>2$ was arbitrary, $0 \in$ $\bigcap_{p>2} S^{p}(Y)$. That 0 is the only point in $\cap_{p>2} S^{p}(Y)$ follows from the construction of $Y$ and the fact that $x \in S^{p}(Y)$ if and only if $x \in S^{p}(Y \cap \overline{\Delta(x, r)})$ for any $r>0$.

Given any compact set $X$ it would be interesting to find necessary and sufficient conditions for $\bigcap_{p>2} S^{p}(X)$ to have positive measure. Lemma (I.2.3) 
implies that a sufficient condition is that there exist a single $g$ which represents 0 for $R^{p}(X)$ for all $p>2$.

\section{BIBLIOGRAPHY}

1. J. Brennan, Point evaluations and invariant subspaces, Doctoral Dissertation, Brown Univ., Providence, R.I., 1968.

2. Invariant subspaces and rational approximation, J. Functional Analysis 7 (1971), 285-310.

3. Andrew Browder, Introduction to function algebras, Benjamin, New York, 1969. MR 39 \#7431.

4. Jacques Deny, Sur la convergence de certaines intégrales de la théorie du potentiel, Arch. Math. 5 (1954), 367-370. MR 16, 589.

5. Herbert Federer, Geometric measure theory, Springer-Verlag, New York, 1969. MR 41 \#1976.

6. W. H. J. Fuchs, Topics in the theory of functions of one complex variable, Van Nostrand, Princeton, N.J., 1967. MR 36 \#3954.

7. T. Gamelin, Uniform algebras, Prentice-Hall, Englewood Cliffs, N.J., 1969.

8. L. I. Hedberg, Approximation in the mean by analytic functions, Trans. Amer. Math. Soc. 163 (1972), 157-171.

9. Bounded point evaluations and capacity, J.' Functional Analysis 10 (1972), 269-280. MR 50 \#4980.

10. L. H. Helms, Introduction to potential theory, Interscience, New York, 1969. MR 41 \#5638.

11. N. S. Landkof, Foundations of modern potential theory, Springer-Verlag, Berlin and New York, 1972. MR 50 \#2520.

12. L. Loomis, An introduction to abstract harmonic analysis, Van Nostrand, Princeton, N.J., 1953. MR 14, 883.

13. A. G. O'Farrell, Analytic capacity, Hölder conditions, and $\tau$-spikes, Trans. Amer. Math. Soc. 196 (1974), 415-424. MR 50 \# 13562.

14. __ An isolated bounded point derivation, Proc. Amer. Math. Soc. 39 (1973), 559-562. MR 47 \# 4001.

15. Walter Rudin, Real and complex analysis, McGraw-Hill, New York, 1966. MR 35 \# 1420.

16. S. O. Sinanjan, Approximation by polynomials and analytic functions in the areal mean, Mat. Sb. 69 (111) (1966), 546-578; English transl., Amer. Math. Soc. Transl. (2) 74 (1968), 91-124. MR 35 \#389.

17. J. Wang, An approximate Taylor's theorem for $R(X)$, Math. Scand. 33 (1973), 343-358. MR 50 \#622.

18. __ Modulus of approximate continuity for $R(X)$, Math. Scand. 34 (1974), 219-225. MR 50 \#7556.

19. J. Wermer, Potential theory, Lecture Notes in Math., vol. 408, Springer-Verlag, New York, 1974.

20. D. R. Wilken, Bounded point derivations and representing measures on $R(X)$, Proc. Amer. Math. Soc. 24 (1970), 371-373. MR 40 \# 1781 .

Department of Mathematics, Brown University, Providence, Rhode Island 02912

Current address: Mathematics Department, East Carolina University, Greenville, North Carolina 27834 